INGENIERÍA QUÍMICA

\title{
Evidencias del choque térmico en partículas de polvo después de una explosión en minería de carbón
}

CHEMICAL ENGINEERING

\section{Heat shock evidences within dust particles after explosion in coal mining}

\author{
Jorge M. Molina-Escobar§*, Astrid Blandón* \\ *Facultad de Minas, Universidad Nacional de Colombia, Sede Medellín \\ sjmmolina@unal.edu.co,asblando@unal.edu.co
}

Recibido: 12 de Diciembre de 2011 - Aceptado 28 de Abril de 2014

\begin{abstract}
Resumen
Con el fin de determinar las temperaturas alcanzadas y el grado de transformación del polvo de carbón durante explosiones en dos minas subterráneas, se tomaron tanto muestras de los mantos de carbón originales, como las resultantes de la explosión del polvo de carbón. Una mina corresponde a la Región 1 donde se explota carbón térmico, y la otra a la Región 2 con explotación de carbón coquizable. Estas muestras se observaron al microscopio en luz reflejada normal y fluorescente; se hicieron secciones pulidas tanto del polvo de carbón como del char obtenido de la desgasificación a $950^{\circ} \mathrm{C}$ efectuada en el laboratorio. Igualmente, se sometieron a microscopía electrónica de barrido con microanálisis de energía dispersiva SEM-EDX para determinar la morfología y la composición química. Se observó el efecto térmico en ausencia de oxígeno no sólo en la materia orgánica, en la cual hay evidencia de la desgasificación y la formación de char y coque, sino también en la materia mineral, en dónde se encontraron minerales fundidos. Los resultados indican que el polvo de carbón de la región 1 en general, es menos explosivo que el de la región 2, igualmente el poder calorífico es un parámetro influyente en la explosividad, más no así la materia volátil. Cada tipo y rango del carbón presentan características muy diferentes frente a la explosividad y por lo tanto no se puede generalizar la influencia de los diferentes factores en este proceso.
\end{abstract}

Palabras claves: Choque Térmico, explosión, mina subterránea, polvo de carbón.

\begin{abstract}
In situ coal samples and coal dust explosion samples were taken with the aim to determine the raised temperatures and the transformation grade of the coal dust during an explosion in two underground mines in Colombia. One of them exploited thermic coal (Region 1) and the other coking coal (Region 2). These samples were observed under the microscope of reflected normal and fluorescent light; some polish sections of coal dust and the char obtained from the degasification at $950^{\circ} \mathrm{C}$ in the laboratory, were observed and also analyzed with scanning electronic microscopy SEM-EDX to determine the morphologic characteristics and chemical composition. The thermic effect was observed in the absence of oxygen in the organic matter, in which is possible to visualize the evidences of degasification and the char and coke formation, as well the mineral matter, with the presence of melted minerals. The results indicate that the coal dusts from the Region 1 are in general fewer explosives than the coal dusts from the Region 2, also the calorific value is a parameter influencing the explosivity, but not so volatile matter. Every type and coal rank presents different characteristics compared to the explosivity and therefore cannot be generalized.
\end{abstract}

Key words: Coal dust, explosion, thermic effect, underground Mine 


\section{Introducción}

Las explosiones no controladas en minas de carbón han sido un problema por más de 200 años. La necesidad de carbón como una de las principales fuentes de energía demanda el incremento de la minería, la cual requiere a su vez, de técnicas mejoradas para la prevención de explosiones de polvo de carbón; para ello, es necesario entender cómo se comporta el polvo de carbón durante una explosión y cómo se puede evidenciar su participación en ella. Una explosión de polvo de carbón, es más compleja que el fuego. Para provocarla se requiere la presencia simultánea de las siguientes seis (6) condiciones: Fuente de inflamación, campo de explosividad, aire, confinamiento, polvos combustibles y polvos en suspensión. (Taborda y Echeverry, 1995; Londoño y Rada 1996, Castro y Martínez, 1997, 1998 y 1999). Dentro de estas condiciones deben estar las que conforman el triángulo del fuego y bien conocidas:

Combustible (carbón y gas).

Comburente (oxígeno).

Fuente de ignición (chispas eléctricas, autocalentamiento y llamas abiertas, gas, voladuras con explosivos no permisibles), entre otros.

Las siguientes condiciones físicas del carbón también intervienen en la inflamación y propagación de nubes de polvo:

La naturaleza del carbón, tal como el rango y la composición maceral.

El tamaño de las partículas (menores a malla 100, Sapko et al., 2007).

La concentración de partículas finas (menores a malla 100).

Según Humphreys y O'Beirne (2000), una explosión de polvo de carbón ocurre cuando partículas finas se ponen en suspensión y empiezan a quemarse por algún medio. En las minas de carbón, el precursor usualmente es el metano o el indebido uso de explosivos. La llama resultante viaja levantando el polvo de carbón en medio de gases calientes, que pueden alcanzar más de 1000 grados centígrados.

Cuando hay un grado de confinamiento en los túneles, los gases no se pueden expandir y se genera una onda de presión hasta de 7 bares, la cual acelera la llama levantando más partículas de carbón y provocando que la explosión continúe; ello genera presiones destructivas y grandes cantidades de gases tóxicos e irrespirables. Finalmente, una explosión de polvo de carbón podría propagarse a lo largo de toda la mina hasta alcanzar la superficie. Para que no se produzca una explosión de polvo de carbón, el solo hecho de eliminar una de las seis condiciones es más que suficiente; no obstante, lo ideal sería eliminar dos. El tema es que el polvo de carbón siempre va a estar presente en las operaciones mineras del arranque y el transporte. Lo que se hace en la práctica es retirarlo de la mina, o mezclar el polvo de carbón con otro polvo incombustible o con agua de tal forma que lo neutralice o lo inertice.

Es común encontrar que una mezcla con un $65 \%$ de material incombustible no produce explosiones, o lo son muy débiles, menores a 2 bares. Este porcentaje normalmente debe ser mayor dependiendo, entre otros, del rango del carbón, poder calorífico, contenido de volátiles y del contenido de metano.

Weiss et al. (1989) encontraron que algunas minas requerían entre 79 y $82 \%$ de material incombustible para evitar las explosiones. Sapko et al. (1989) confirmaron que para polvo fino de carbón, la mina de Lake Lynn ubicada en Fayette, Pensilvania, Estados Unidos, requería más del 70\% de material incombustible con un carbón del 18\% de materia volátil. En este artículo debe entenderse el grado de explosividad del polvo de carbón, como el porcentaje de material incombustible que se requiere para su neutralización. Así, un polvo de carbón que requiera del $95 \%$ de polvo incombustible, será más explosivo que aquel que sólo requiera un $80 \%$. 
Se sabe que las partículas de carbón se queman de una manera compleja. El proceso de combustión incluye reacciones superficiales heterogéneas, desvolatilización y subsecuentes reacciones de los componentes volátiles, hinchamiento, fracturamiento y otros cambios fisicoquímicos que ocurren en las partículas. Además, se sabe que las partículas de carbón no siempre se queman simplemente como esferas, sino que también se pueden quemar internamente y formar cenosferas (esferas huecas). La composición de la materia volátil desprendida y la combustión en sí misma, depende de la tasa de calentamiento de la llama, haciendo las descripciones analíticas del proceso extremadamente difíciles (Krazinski et. al., 1979).

Un punto importante a tener en cuenta es que un confinamiento parcial de una nube de polvo iniciada es suficiente para producir una explosión altamente dañina. En este sentido, muchas nubes de polvo, se comportan similar a nubes de gases y vapores inflamables, (Proust C. 2005).

A raíz de las explosiones de los últimos años en Colombia, se tomaron muestras de polvo en diferentes partes de las minas con el fin de detectar la presencia de partículas quemadas o afectadas por el choque térmico y tratar de establecer la participación del polvo de carbón, determinando las temperaturas alcanzadas durante las explosiones. Se realizaron análisis petrográficos, próximos y se hicieron calentamientos a diferentes temperaturas a muestras originales del depósito y se compararon con las tomadas después de la explosión.

\section{Metodología}

Se tomaron aproximadamente 20 muestras del polvo resultante en la explosión de polvo de carbón en diferentes puntos de una mina en donde se explota carbón térmico de la Región 1, y otra donde se explota carbón coquizable de la Región 2. De igual manera, se recolectaron muestras in-situ de carbón en cada una de las regiones estudiadas. Estas muestras se caracterizaron mediante análisis próximo y de explosividad.

Las muestras de polvo resultantes de la explosión se observaron directamente al microscopio en luz reflejada, transmitida y fluorescente; posteriormente se montaron en resina y se hicieron los pulidos correspondientes. Las muestras se sometieron a microscopía electrónica de barrido con microanálisis de energía dispersiva SEM-EDX para determinar su morfología y composición química.

De otro lado se tomó una muestra de carbón bituminoso alto en volátiles $\mathrm{C}$ y en el horno de caracterización de materia volátil se sometió a un incremento de temperatura durante siete (7) minutos, con el fin de compararla con lo observado en las muestras de polvo obtenidas luego de la explosión en las minas.

\section{Resultados y discusión}

En la figura 1 se muestran las principales características morfológicas del polvo de carbón observado directamente al microscopio en luz reflejada normal y luz reflejada fluorescente, ligado al proceso de transformación de la materia orgánica y los minerales durante una explosión en una mina subterránea.

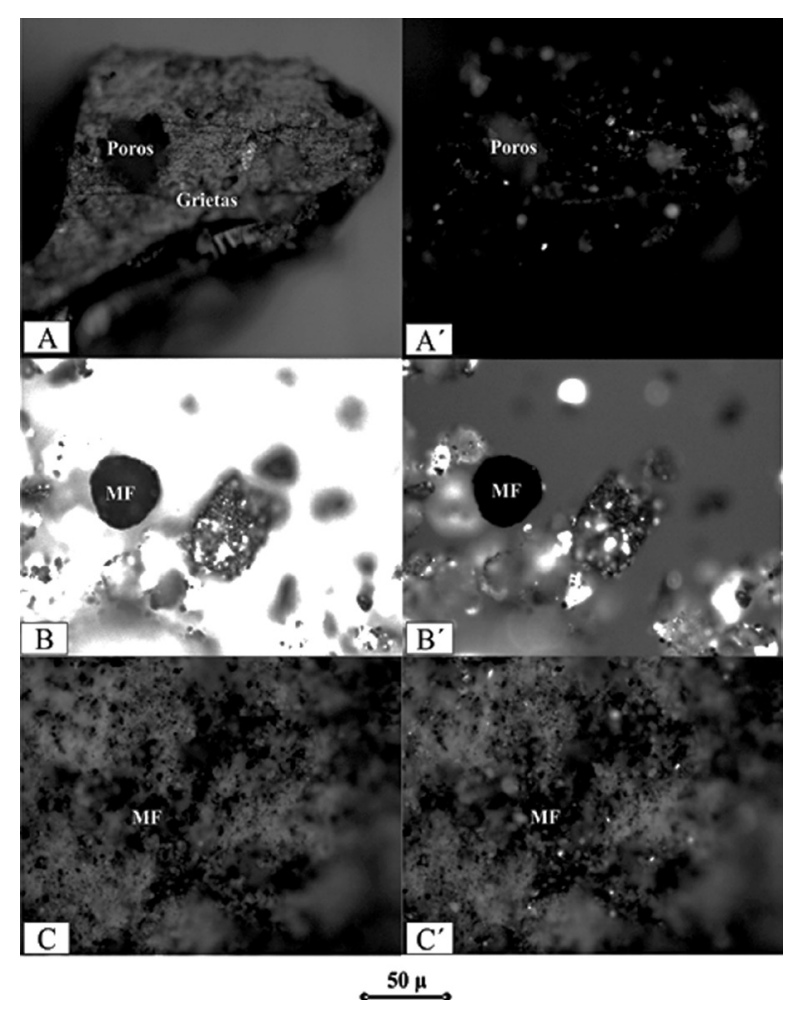

Figura 1. Descripción microscópica en luz reflejada normal $A, B$ y $C$, y luz reflejada fluorescente $A^{\prime}, B^{\prime}, y C^{\prime}$ del proceso de transformación de la materia orgánica como resultado de una explosión de polvo de carbón en una mina subterránea $\mathbf{M F}=$ Minerales fundidos 


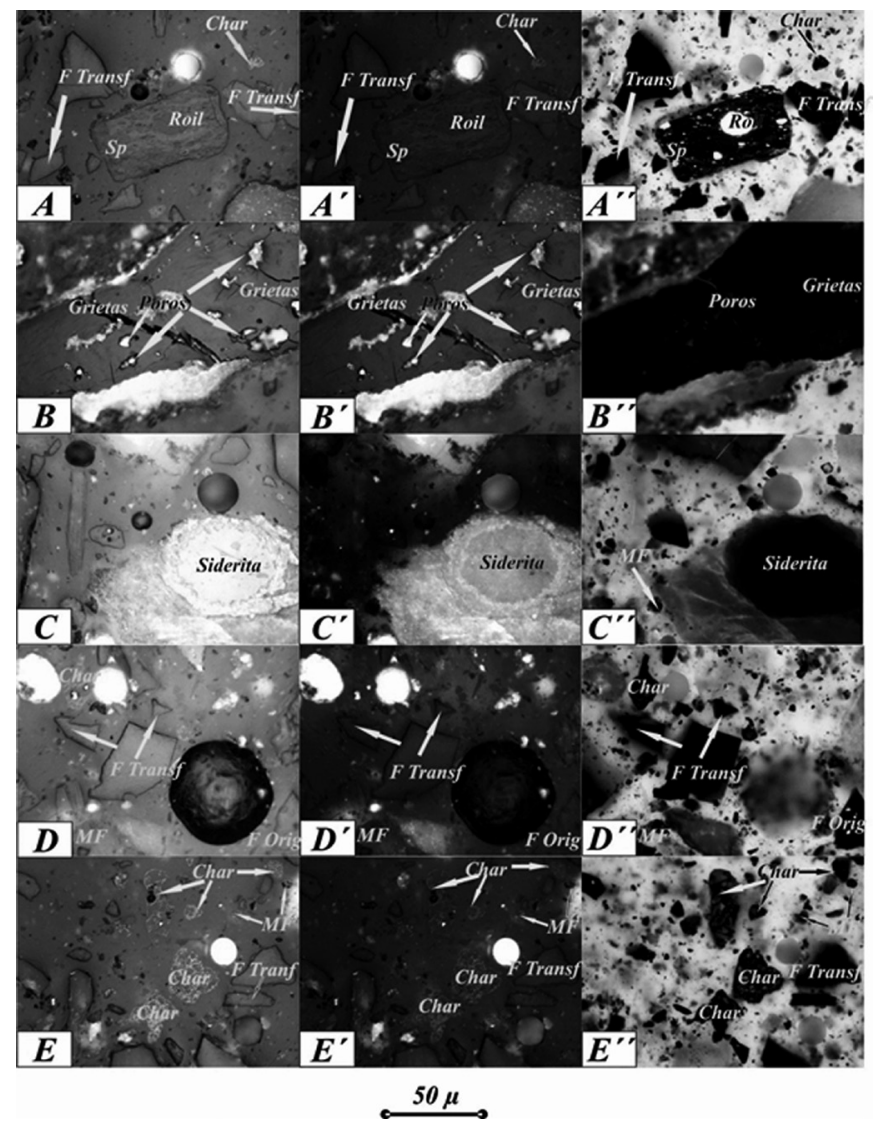

Figura 2. Descripción microscópica en luz reflejada normal $A, B, C$. D y E. Nicoles cruzados, $A^{\prime}, B^{\prime}, C^{\prime}, D^{\prime}$, y $E^{\prime}$, y luz reflejada fluorescente $A^{\prime \prime}, B^{\prime \prime}, C^{\prime \prime}, D^{\prime \prime}$ y $E^{\prime \prime}$, del proceso de transformación de la materia orgánica como resultados de una explosión de polvo de carbón. $F$ Transf $=$ Fragmentos parcialmente desgasificados, $R$ oil $=$ Resinita Oil, $S p=$ Esporinita, $M F=$ Minerales fundidos, $F$ orig = Fragmentos originales.

En las figuras $1 \mathrm{~A}$ y $1 \mathrm{~A}^{\prime}$, se observa una partícula de carbón con poros y grietas como resultado de la desgasificación del carbón al aumentar la temperatura por encima de los $1000^{\circ} \mathrm{C}$, se observa que a $950^{\circ} \mathrm{C}$ es menor el efecto. En las figuras 1B, y 1B'; se observan partículas de diferente tamaño, en donde se destaca el redondeamiento de varias de ellas, indicando un proceso de fusión y posterior re-solidificación. Y en las figuras $1 \mathrm{C} \mathrm{y}$ $1 \mathrm{C}^{\prime}$, se evidencia una masa de minerales fundidos de tamaño de partícula pequeño.

\subsection{Descripción microscópica}

Por medio de la técnica de microscopía óptica se analizó el montaje en sección pulida del polvo de carbón recolectado luego de la explosión en una mina. La observación se efectuó en luz reflejada normal, nicoles cruzados y luz reflejada fluorescente del proceso de transformación de materia orgánica y minerales, tal como se observa en la Figura 2. En las figuras 2A, $\mathrm{A}^{\prime}$ y $\mathrm{A}^{\prime \prime}$ se puede observar una partícula de carbón sin transformar donde se aprecian los macerales característicos de estos carbones subbituminosos, tales como: vitrinita y liptinita, donde estas últimas aparecen representadas por esporinitas y resinita oil (gotas de aceite). Se tienen fragmentos parcialmente desgasificados (se pierde el color de fluorescencia) y fragmentos totalmente desgasificados (char). Las figuras 2B, B'y B" evidencian una partícula de carbón en proceso de transformación. Se puede observar el agrietamiento de la partícula y la presencia de poros, en donde aparecen coloraciones similares a las que presentan los minerales, pero que en realidad es el efecto de las reflexiones de la luz. En las figuras $2 \mathrm{C}, \mathrm{C}^{\prime}$ y $\mathrm{C}^{\prime \prime}$, se presenta la manera como aparecen los carbonatos (concreciones de siderita, las cuales son comunes en los carbones 
de la zona 1) y la apariencia que toman cuando se someten a temperaturas por encima de los $1000^{\circ} \mathrm{C}$. También se observan pequeñas partículas redondeadas que corresponden a minerales. En las figuras 2D, D' y $\mathrm{D}^{\prime \prime}$, se observan diferentes tipos de granos tales como fragmentos de materia orgánica parcialmente desgasificados, pequeñas partículas de minerales fundidos de forma esférica, fragmentos de diferentes formas y tamaños de materia orgánica totalmente desgasificada (char) $\mathrm{y}$ algunos fragmentos originales. Las figuras $2 \mathrm{E}$, E'y E" muestran partículas de char de diferentes formas y tamaños, en dónde se evidencia la total desgasificación de la materia orgánica, pequeñas partículas esféricas de minerales fundidos y fragmentos de materia orgánica parcialmente desgasificada.

De acuerdo con el contenido de materia volátil libre de agua y ceniza, el carbón de la Región 1 corresponde a un carbón Bituminoso Alto en Volátiles $\mathrm{C}$, con una humedad que varía entre 11 y $13 \%$; su contenido de cenizas es de 2.3 a $3.7 \%$ y su contenido de materia volátil está entre 40 y
$48 \%$. Esto no se encuentra registrado claramente en una tabla.

Los resultados anteriores verifican la variación vertical en la composición del manto, lo cual está ligado a la variación en la susceptibilidad a la combustión espontánea y por lo tanto también al contenido de gases en el carbón (Caro L., 2013.)

La figura 3 muestra las características originales del carbón térmico de la Región 1 . Aquí se puede observar el bajo grado de transformación de la materia orgánica; se reflejan claramente los tres grupos macerales:

1. Grupo de vitrinita. Presenta colotelinita $(\mathrm{Ct}) \mathrm{y}$ colodetrinita $(\mathrm{Cd})$.

2. Grupo de liptinita. Se observan macerales tales como esporinitas $(\mathrm{Sp})$, cutinitas $(\mathrm{Cu})$, liptodetrinitas (Lp), exsudatinitas (Ex), etc.

3. Grupo de la inertinita. Dentro de los macerales se tiene la funginita $(\mathrm{Fg})$.

La Figura 4 presenta la morfología y la composición

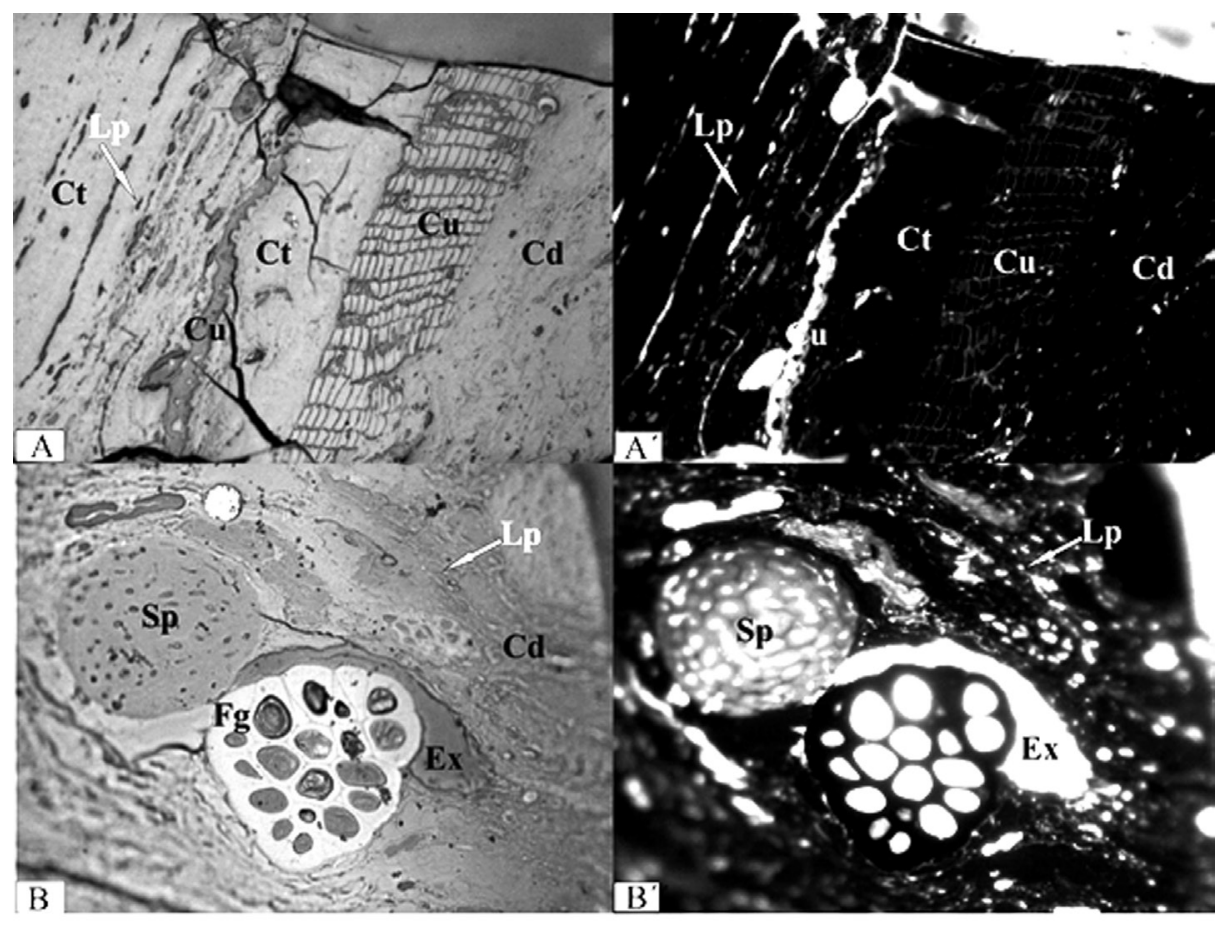

$\stackrel{50 \mu}{\longrightarrow}$

Figura 3. Características de los macerales de la muestra original del carbón de la Región 1 A. B, luz reflejada normal, $A^{\prime}, B^{\prime}$, Luz reflejada fluorescente.

$C t=$ Colotelinita, $C d=$ Colodetrinita, $S p=$ Esporinita, $C u=$ Cutinita, L $p=$ Liptodetrinita, $E x=$ exsudatinita,,$F g=$ Funginita . 


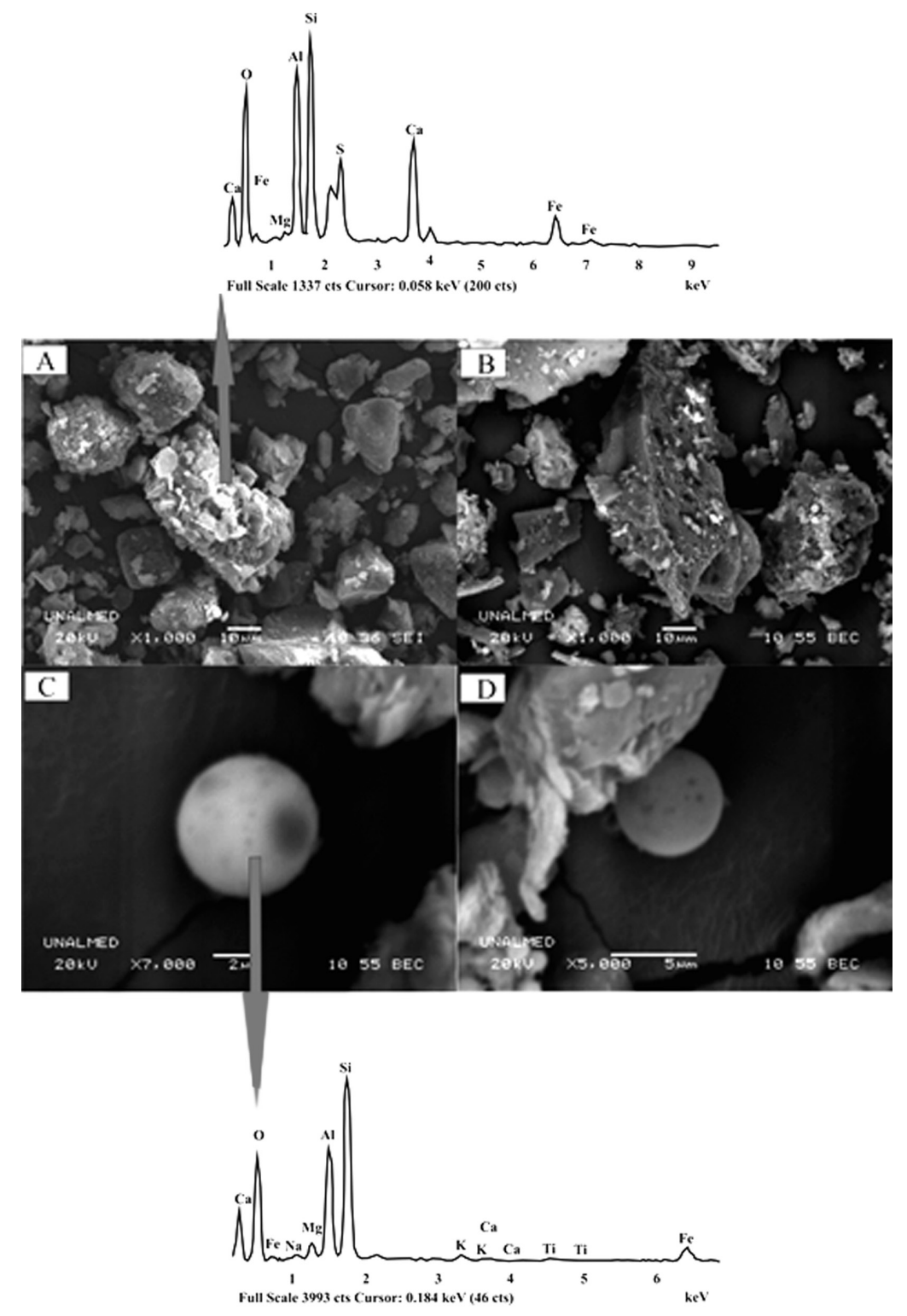

Figura 4. Morfología y composición química obtenida mediante microscopía electrónica de barrido SEM-EDX durante una explosión de polvo de carbón en una mina subterránea en dónde se explota carbón térmico de bajo rango de la Región 1

química obtenidas mediante la técnica SEMEDX, después de la explosión de polvo de carbón en una mina subterránea en dónde se explota carbón térmico de bajo rango (bituminoso alto en volátiles $\mathrm{C}$ ) de la Región 1. Aquí se observan minerales tales como silicatos, figura $4 \mathrm{~A}-4 \mathrm{D}$ y partículas redondeadas de silicatos compuestos principalmente por óxidos de silicio, aluminio y calcio, Figura 4C y 4D. Se observan poros y grietas como resultado de la desgasificación de la muestra. La figura 5 muestra las características originales del carbón coquizable de la Region 2. Aquí se puede observar el alto grado de transformación de la materia orgánica, colotelinita $(\mathrm{Ct})$, exsudatinita (Ex), semifusinita $(\mathrm{Sm})$, y los minerales más comunes presentes en la muestra como son la pirita framboidal (Py) y los carbonatos (Ca).

En la figura 6, se pueden observar la morfología y la composición química del polvo resultante luego de la explosión en una mina subterránea de la Región 2, en donde se explotan carbones 


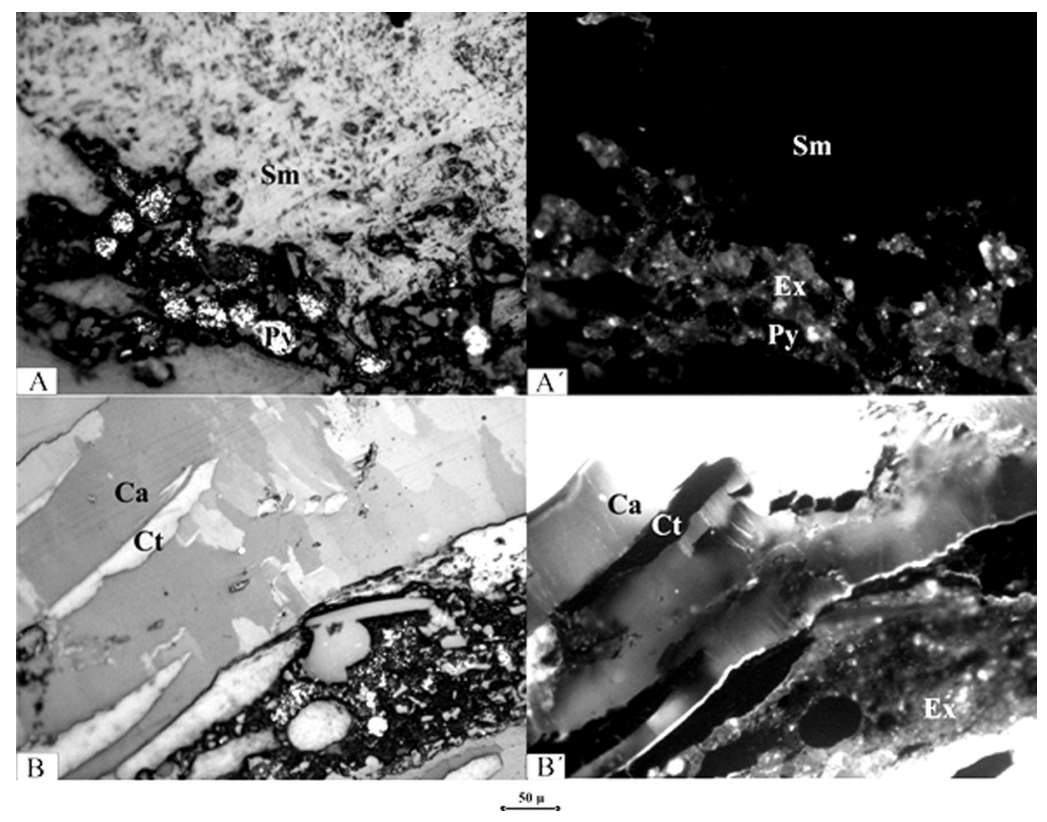

Figura 5. Caracteristicas de los macerales de la muestra original del carbón de la Región 2. A, B. luz reflejada normal, $A^{\prime}, B^{\prime}$ Luz reflejada fluorescente $C t=$ Colotelinita,$E x=$ exsudatinita, $S m=$ Semifusinita, $P y=$ Pirita, $C a=$ Carbonatos.

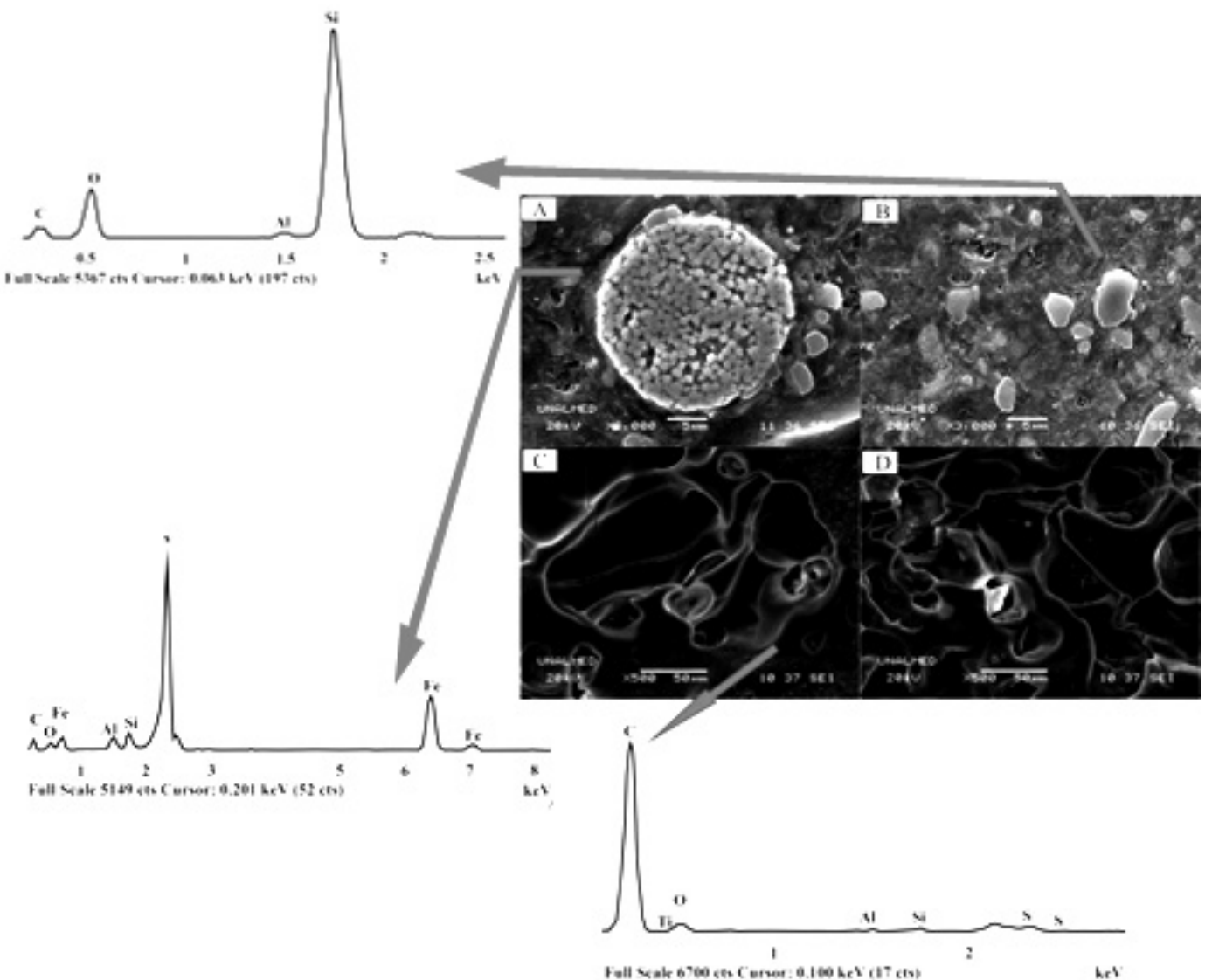

Figura 6. Características y composición química obtenida mediante microscopía electrónica de barrido SEM-EDX durante una explosión de polvo de carbón en una mina subterránea en dónde se explota carbón coquizable de la Región 2 
coquizables. La figura 6A, presenta la pirita framboidal (sulfuro de hierro) y algunos poros ocasionados por la desgasificación de la muestra. En la figura 6B, se observan unas partículas redondeadas de cuarzo (óxido de silicio), e igualmente, se ven poros como resultado de la desgasificación de la muestra. En las figuras 6C y $6 \mathrm{D}$, se nota claramente la transformación de la materia orgánica y se evidencia la plasticidad de los macerales, el tamaño y forma de los poros, al igual que el fisuramiento. En dichas figuras también se observa que la matriz está enriquecida en carbono como producto de la transformación térmica. Estas características son típicas de la materia orgánica de un carbón con propiedades plásticas, cuando se somete a temperaturas mayores de $1000^{\circ} \mathrm{C}$. De otro lado, mediante la observación a una sección pulida de una muestra de carbón de la Región 1 sometida a $950^{\circ} \mathrm{C}$ durante 7 minutos, se pudo observar la transformación de los macerales vitriníticos y liptiníticos, dejando un char grueso con poros irregulares de todas las formas y tamaños, al igual que muchas grietas, tal como se evidencia en las figuras 7A y B. A diferencia de las muestras obtenidas después de la explosión en la mina de la Región 1, el char es mucho más delgado y con poros redondeados mejor definidos (figura 2E), lo cual indica que la temperatura alcanzada durante la explosión del polvo de carbón en la mina subterránea de la región 1 , fue muy superior a los $950^{\circ} \mathrm{C}$. De todas las descripciones anteriores, se puede decir que hay una gran similitud entre el comportamiento de los minerales sometidos a altas temperaturas (esféricos) y las partículas de carbón (porosas y con grietas), con los observados en las muestras sometidas a $950{ }^{\circ} \mathrm{C}$ durante 7 minutos. Posiblemente esto indica que la explosión alcanzó temperaturas por encima de $\operatorname{los} 1000^{\circ} \mathrm{C}$ en dónde hubo participación activa del polvillo de carbón, dado que este fue transformado y, probablemente, asociado a metano y otros gases como el $\mathrm{CO}_{2}$.

En la figura 8 se encuentran las diferencias entre los análisis próximos y de explosividad en las dos muestras de polvo de carbón; en la Región 1: carbones térmicos, y en la Región 2: carbones con propiedades plásticas. Las muestras de la Región 1 presentan características totalmente contrarias a las de la Región 2, tales como: mayores porcentajes de humedad $(4,72-$ $13,99 \%)$ y contenidos de materias volátiles

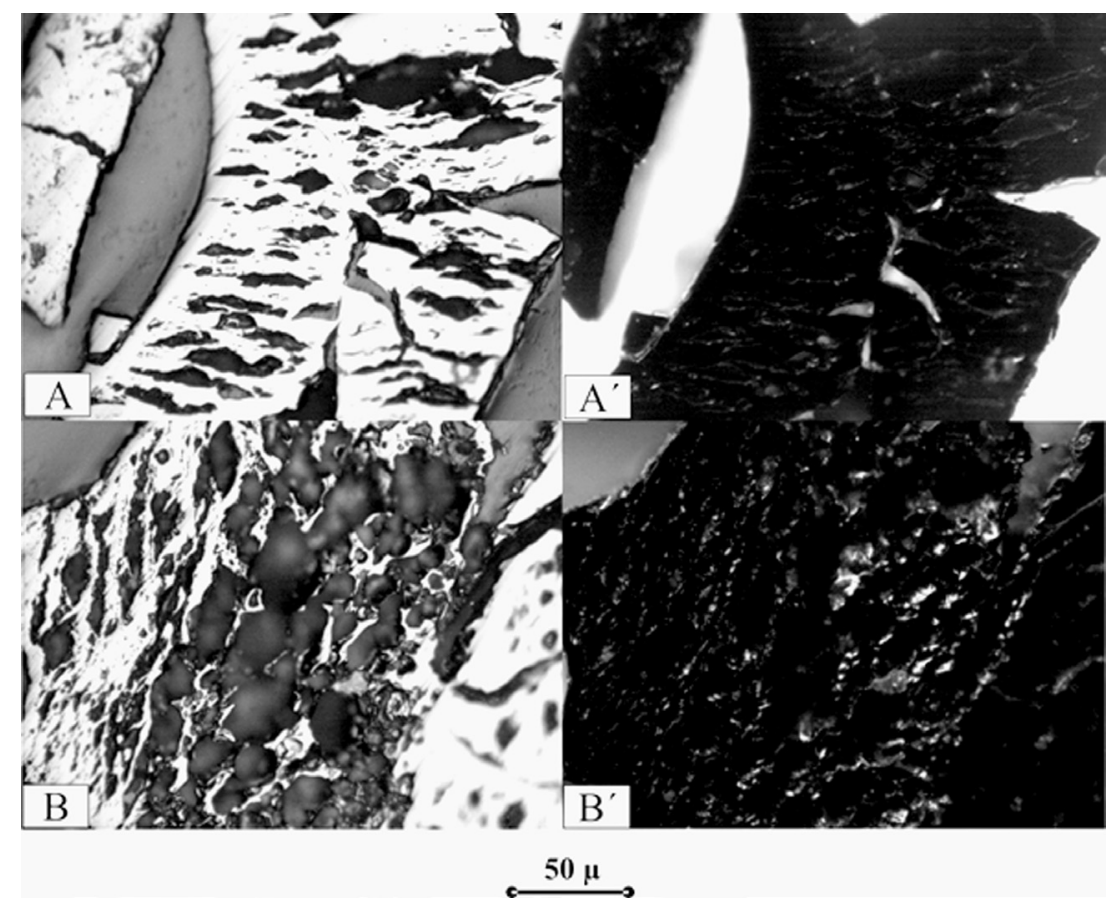

Figura 7. Char obtenido a $950^{\circ} \mathrm{C}$, A y B Luz reflejada normal; $A^{\prime}$ y $B^{\prime}$. Luz reflejada fluorescente 


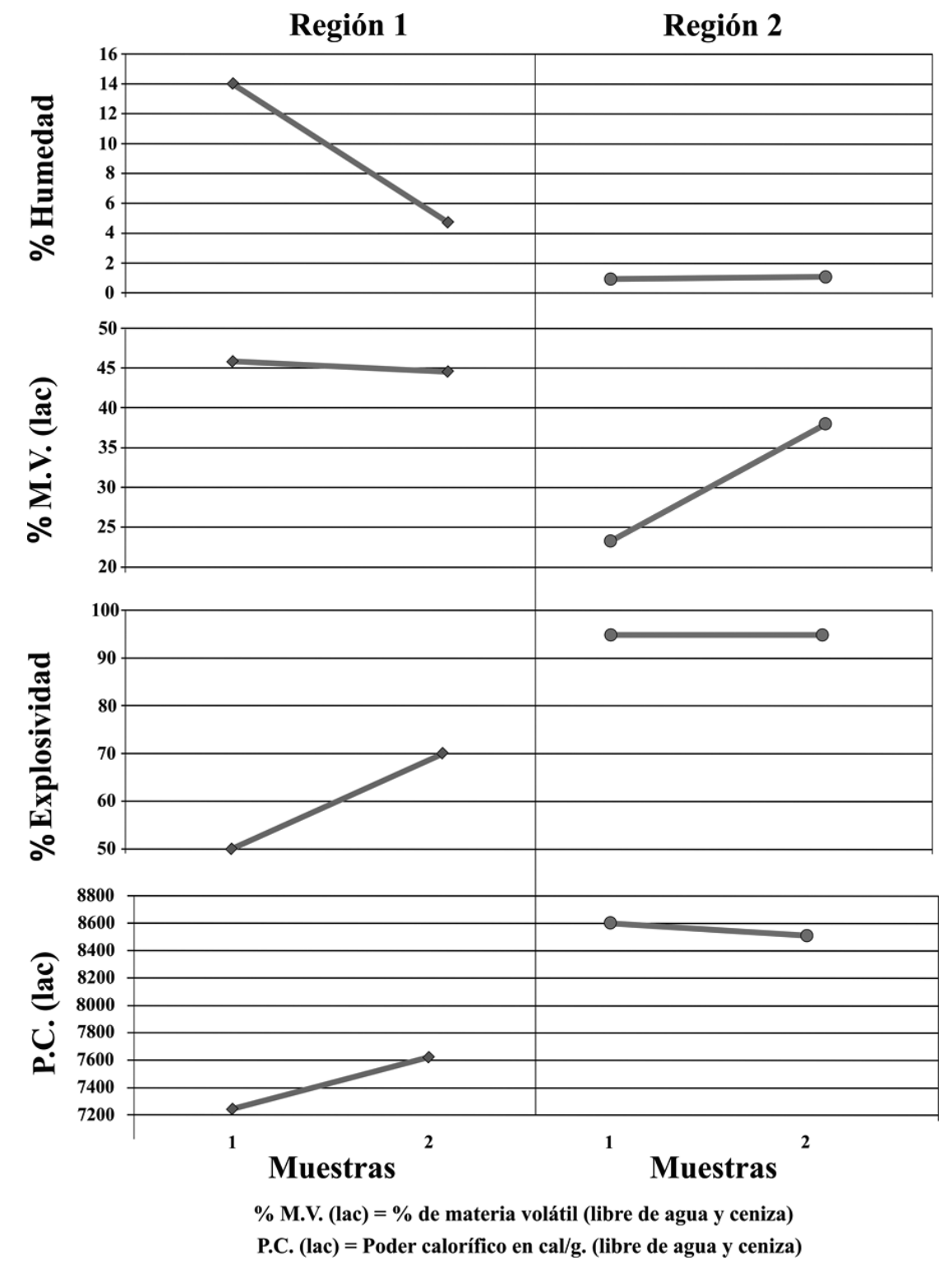

Figura 8. Resultados de los análisis de humedad, materia volátil, poder calorifico y explosividad de muestras de polvo de carbón de dos minas en la Región 1 y dos en la Región 2

libres de agua y cenizas, (45,77 - 44,53); menor poder calorífico libre de agua y ceniza $(7243-7620 \mathrm{cal} / \mathrm{g}) ; \mathrm{y}$ menores porcentajes de explosividad $(50-70 \%)$. Sin embargo, dentro de la Región 1 hay una relación directa entre el porcentaje de explosividad y el poder calorífico; es decir, a mayor poder calorífico libre de agua y cenizas, mayor porcentaje de explosividad del polvo de carbón. También presenta una relación inversa entre la humedad y las materias volátiles con el porcentaje de explosividad, así que a mayor humedad y materia volátil menor porcentaje de explosividad. Para el caso de la Región 2, aunque existe variación importante en el contenido de materias volátiles libres de agua y ceniza $(23,36-38,05 \%)$, no existen diferencias significativas en los porcentajes de humedad (0.96 - 1.09), poder calorífico (8506 $8601 \mathrm{cal} / \mathrm{g})$ y explosividad (95\%).

De esta manera y de acuerdo con los resultados de la tabla 1 , se requiere mayor cantidad de polvo de roca para inertizar el polvo de carbón generado en una mina de la Región 2, frente al necesario para 
Tabla 1. Resultados de los análisis próximos y de explosividad de dos muestras de carbón de la Región1 y dos muestras de carbón de la Región 2

\begin{tabular}{|c|c|c|c|c|c|c|c|c|c|}
\hline Muestra & $\begin{array}{l}(\%) \\
H R\end{array}$ & $\begin{array}{c}(\%) \\
C z\end{array}$ & $\begin{array}{c}(\%) \\
M . V .\end{array}$ & $\begin{array}{l}\text { MV } \\
\text { (lac) }\end{array}$ & $\begin{array}{l}(\%) \\
C . F\end{array}$ & $\begin{array}{l}(\%) \\
S T\end{array}$ & $\begin{array}{l}P . C . \\
\mathrm{cal} / \mathrm{g}\end{array}$ & $\begin{array}{c}P . C . \\
\mathrm{cal} / \mathrm{g} \\
(\mathrm{lac})\end{array}$ & Explosividad \\
\hline Región 1 (1) & 13,99 & 6,62 & 36,34 & 45,77 & 43,05 & 0,62 & 5750 & 7243 & 50 \\
\hline Región 1 (2) & 4,72 & 6,03 & 39,74 & 44,53 & 49,51 & 0,71 & 6801 & 7620 & 70 \\
\hline Región 2 (1) & 0,96 & 6,35 & 21,65 & 23,36 & 71,04 & 0,53 & 7972 & 8601 & 95 \\
\hline Región 2 (2) & 1,09 & 4,57 & 35,9 & 38,05 & 58,44 & 0,78 & 8025 & 8506 & 95 \\
\hline
\end{tabular}

evitar la explosión en la Región 1.

No obstante, no se puede generalizar cuando se está determinando el porcentaje de explosividad de los carbones, ya que cada tipo y rango del carbón presentan características muy diferentes. Así por ejemplo los carbones de la Región 1, normalmente presentan menor rango, menor grado de explosividad y menor poder calorífico, y a su vez, mayor humedad y mayor contenido de materias volátiles. Por el contrario los carbones de la Región 2 , son de mayor rango, tienen menor contenido de humedad y materia volátil, pero presentan un alto poder calorífico y alto grado de explosividad, tal como se puede observar en la figura 8 .

La misma figura permite evidenciar como el poder calorífico se constituye en un importante parámetro para favorecer la separación de los carbones más propensos a la explosividad. Así y como era de esperarse, los carbones de menor poder calorífico presentan menor explosividad.

La materia volátil no se puede utilizar indistintamente, pues depende del tipo de carbón. Así dentro de los carbones térmicos, el que presenta menor contenido de materias volátiles, asociado a un mayor rango es el que tiene mayor explosividad. Contrario a ello, en los carbones con propiedades plásticas esto no se cumple, aunque hay variaciones en el contenido de materias volátiles no se ve reflejado en la explosividad.

\section{Conclusiones}

Las explosiones de metano y polvo de carbón o combinaciones de éstos, alcanzan temperaturas superiores a $1000^{\circ} \mathrm{C}$. En este estudio se analizaron diversas muestras resultantes de explosiones que se presentaron en dos minas subterráneas de dos regiones diferentes en Colombia y al confrontarlas con muestras de los mantos de carbón originales, reflejan el mismo comportamiento al ser sometidas a condiciones de laboratorio; esto es, pequeños minerales redondeados, aparición de chars, coque, grietas, poros y cavidades por la disminución de volátiles.

Se ratifica una vez más, como las técnicas de caracterización incluyendo la petrografía son de gran ayuda, no sólo para conocer previamente los componentes orgánicos y minerales sino también para entender cómo se comportan y transforman dichos componentes al someterlos a altas temperaturas durante cortos periodos de tiempo, como sucede en una explosión de polvo de carbón.

Se encontró un comportamiento diferente al comparar los carbones térmicos y coquizables con respecto a la explosividad, donde los primeros son menos explosivos que los segundos. Lo anterior debido a que normalmente los carbones coquizables son más blandos y por lo tanto generan partículas de polvo más finas, las cuales se encienden rápidamente; además porque en el proceso de transformación de la materia orgánica se generan aceites que se pueden encender más fácil y alcanzan una mayor temperatura. (Baquero, Blandón y Molina, 2012).

Un parámetro que ayuda en la clasificación de los carbones más propensos a la explosividad es el poder calorífico, cuando se trata de polvos de carbón provenientes de mantos de la misma región. Así se demostró que hay una relación directa entre 
el poder calorífico y el grado de explosividad, de tal modo que a mayor poder calorífico, mayor grado de explosividad.

La materia volátil no se puede utilizar indistintamente para conocer el grado de explosividad de los carbones, dado que ello depende del tipo de carbón. Así, para el caso de carbones coquizables el contenido de materia volátil no es un factor determinante, ya que en este caso es más importante el tamaño de grano de las partículas de polvo y el poder calorífico.

No se puede generalizar cuando se está determinando el porcentaje de explosividad de los carbones, ya que cada tipo y rango del carbón presentan características muy diferentes, en cuanto a dureza, poder calorífico, humedad, contenido de cenizas, entre otros.

También se concluye que después de una explosión en una mina de carbón, si al analizar los polvos remanentes y se encuentra que éstos han sido alterados o transformados, si se evidencia la presencia de char o minerales fundidos, entre otros, es porque hubo participación del polvo de carbón como parte del combustible de la explosión y se lograron temperaturas por encima de $\operatorname{los} 950^{\circ} \mathrm{C}$.

\section{Referencias bibliográficas}

Baquero et al. (2012). Análisis de los factores que influyen en la explosividad del polvo de carbón en las minas subterráneas. Revista Ingeniería y Competitividad 14, (2), 147-160.

Caro L., (2013). Evaluación de las asociaciones de litotipos en la generación y almacenamiento de Gas Asociado al Carbón (GAC) en los carbones de la Formación Amagá entre los municipios de Amagá y Angelópolis. Universidad Nacional de Colombia Sede Medellín. Facultad de Minas. 148.

Castro W \& Martínez C (1997). Combustión espontánea en macizos de carbón. Revista DYNA, (123), 43-50.

Castro W \& Martínez C (1998). Riesgo potencial de explosiones de polvo combustible en minas subterráneas de carbón. Revista DYNA, (124), 37-53.
Castro W \& C Martínez (1999). Prevención de golpes de polvo en minas de carbón mediante la neutralización con material estéril. Revista DYNA, (126), 43-59.

Humphreys \& O’Beirne (2000). Risk assessment based stone dusting and explosion barrier requeriments, Proc. Queensland mining industry health and safety conference, Townsville, Queensland, Australia: http://www.qmc.com.au/ docs/conferences/QMC 2000/conf humphreys.pdf

Krazinski J. L., Buckius R. O. \& Krier H. (1979). Coal dust flames: A review and development of a model for flame propagation. Progress in Energy and Combustion Science (5), 31-71.

Londoño \& Rada (1996). Riesgo potencial de explosiones de polvo combustible en la cuenca Amaga Angelópolis. Proyecto de Grado en Ingeniería de Minas y Metalurgia, Universidad Nacional de Colombia, Sede Medellín. Facultad de Minas. 181.

Proust, C. (2005). A few fundamental aspects about ignition and flame propagation in dust clouds. J. Loss Prevent. Process Ind. (19), 104-120.

Sapko, M., Cashdollar, K., Gree, G.(1989).Prevention and suppression of coal mine explosions. In Proc. 23rd Int. Conf. of safety in mines research institutes. Washington, DC., Pittsburgh, PA: U.S. Department of the Interior, Bureau of Mines, p. 791-807.

Sapko et al. (2007). Coal dust particle size survey of U.S. mines. J Loss Prev Process Ind, (1), 616-620.

Taborda \& Echeverri (1995). Incendios en macizos de carbón en la cuenca carbonífera Amagá Angelópolis. Universidad Nacional de Colombia, Sede Medellín. Facultad de Minas. 173.

Weiss et al. (1989). Recent results of dust explosion studies at the Lake Lynn experimental mine. Proc. 23rd Int. Conf. of safety in mines research institutes. Washington, D.C., Pittsburgh, PA: U.S. Department of the Interior, Bureau of Mines, p. 843-856. 